\title{
CareLoc: Uma Aplicação de Localização de Pacientes usando Beacons em Ambiente Domiciliar
}

\author{
Matheus S. Rodrigues ${ }^{1}$, Sérgio T. Carvalho ${ }^{1}$ \\ ${ }^{1}$ Instituto de Informática - Universidade Federal de Goiás (UFG) - Goiás - Brasil \\ matheus_silva@discente.ufg.br, sergiodinf.ufg.br
}

\begin{abstract}
One consequence of the increasing elderly population in Brazil and the demand for specific care with this age group is the creation of remote patient monitoring applications. This class of applications has the potential to monitor important patient care information in the home environment, such as physiological data and location. This paper describes the results of a survey conducted to identify existing indoor location methods and presents a mobile application designed to handle user position monitoring and establishment using Bluetooth Low Energy beacons (BLE).
\end{abstract}

Resumo. Uma consequência do aumento da população idosa no Brasil e da demanda de cuidados específicos com esse grupo etário é a criação de aplicações de monitoramento remoto de pacientes. Essa classe de aplicações tem o potencial de monitorar informações importantes para o cuidado do paciente em ambiente domiciliar, como seus dados fisiológicos e sua localização. Este artigo descreve os resultados de uma pesquisa de iniciação científica conduzida a fim de identificar os métodos de localização interna (indoor) existentes e apresenta uma aplicação móvel criada para lidar com o monitoramento e o estabelecimento da posição do usuário por meio da utilização de beacons Bluetooth Low Energy (BLE).

\section{Introdução}

De acordo com o Instituto Brasileiro de Geografia e Estatística (IBGE), a população idosa no Brasil (pessoas com 65 anos ou mais) aumentará de 9,52\% em 2019 para 19,58\% até 2045 [IBGE ]. Este crescimento faz com que a demanda pela preservação da saúde e do bem-estar do idoso também aumente. Neste contexto, desenvolver propostas que auxiliem na manutenção e melhora da saúde mental e física deste grupo etário se torna essencial.

Além da emergente necessidade de propor soluções que auxiliem na saúde geral do idoso, a questão dos cuidadores em si, que em sua maioria no Brasil é formada por familiares, e a prevalência de cuidados domiciliares com idosos não podem ser deixadas de lado [Del Duca et al. 2010]. Sendo assim, muitas soluções são propostas neste contexto [Trinugroho et al. 2012, Le et al. 2008, Trinugroho et al. 2011, Nourizadeh et al. 2009]. Para estas aplicações, ferramentas que possibilitem o monitoramento de dados fisiológicos do paciente, tais como pressão arterial e frequência cardíaca, além da sua localização, são assuntos bastante investigados no contexto das aplicações de monitoramento remoto de pacientes domiciliares [Oliveira and Carvalho 2017, Dickinson et al. 2016, Memon et al. 2017].

Sistemas de Monitoramento Remoto de Pacientes (SMRP), assim como seus módulos, precisam entregar de forma precisa, confiável e íntegra as informações por eles coletadas. Nesse sentido, uma solução de localização de um SMRP deve atender a tais condições oferecendo precisão quanto à posição do paciente e quanto à comunicação. Uma forma de lidar com isso é a implantação de comunicação em tempo real nos SMRP e seus módulos. O objetivo deste 
artigo é identificar os métodos de localização interna disponíveis que utilizam beacons $B L E$, por meio da condução de uma revisão sistemática, e apresentar o CareLoc, solução capaz de localizar em tempo real pacientes em ambientes domiciliares.

Este artigo está estruturado em outras 4 seções: a segunda seção que descreve os conceitos necessários para a implementação da solução CareLoc, a terceira seção que descreve como a solução CareLoc funciona, a quarta seção que descreve a validação da solução através de testes empíricos, e por fim, a última seção que apresenta as considerações finais.

\section{Fundamentação Teórica: Bluetooth Low Energy}

Um beacon BLE consiste em um chipset, uma bateria e uma antena. Trata-se de um dispositivo de transmissão sem qualquer necessidade de conexão à internet, que emite com periodicidade pacotes bluetooth com informações que podem ser usadas pelos receptores em intervalos regulares de tempo [Cay et al. 2017].

Para usar o beacon BLE como ferramenta de localização indoor, os valores do Received Signal Strength Indicator (RSSI) são utilizados. Estes valores representam a potência do sinal emitido pelo beacon e capturado pelo receptor. Quanto mais longe o receptor está do transmissor, mais fraco é este sinal, possibilitando estabelecer uma relação entre a distância e os valores de RSSI. Beacons BLE foram utilizados na solução CareLoc por apresentarem a melhor relação entre precisão e custo [Cay et al. 2017].

\subsection{Métodos de localização}

Para caracterizar e compreender o problema de localização de pacientes em ambientes domiciliares, foi realizada uma Revisão Sistemática da Literatura (RSL) com o objetivo de identificar as técnicas, métodos e algoritmos utilizados para realizar a localização utilizando beacons BLE. Tal revisão foi produzida com base nos métodos propostos por [Keele et al. 2007].

A base da revisão sistemática é o protocolo, definido a partir da seguinte questão de pesquisa: Quais são os métodos, técnicas ou algoritmos utilizados para realizar a localização de pacientes em ambientes indoor utilizando beacons BLE ? A partir desta questão a seguinte string de busca foi definida:

("Indoor Positioning"OR "Indoor Localization") AND ("Beacon"OR "Beacons"OR "Bluetooth Low Energy"OR "BLE")

Esta string foi aplicada nas bases IEEE, ACM e Science Direct, resultando no retorno de 395 artigos. A aplicação dos critérios de elegebilidade e da análise qualitativa foi feita, resultando em 22 artigos aceitos.

Com a leitura completa dos 22 artigos, os seguintes resultados foram obtidos: i) os métodos apresentados na literatura podem ser divididos em quatro grupos, sendo eles: Trilateração, Reconhecimento de padrões (RP), Proximidade e Probabilístico; ii) os métodos utilizam a potência de sinal emitida pelos beacons BLE para estabelecer uma relação entre a potência e a distância; iii) os métodos sofrem de um mesmo problema: a falta de precisão quando são aplicados em ambientes dinâmicos, ou seja, ambientes que possuem uma alta taxa de movimentação de pessoas ou objetos. Mais detalhes à respeito da condução da revisão sistemática podem ser encontrados em [Rodrigues and Carvalho 2019].

\subsection{Comunicação em tempo real}

A implementação do CareLoc utilizou o conjunto de API's (Application Programming Interface) do WebRTC para realizar a comunicação em tempo real entre dois pares na rede. Esta 
ferramenta é uma das mais utilizadas envolvendo aplicações Web e dispositivos móveis, pois permite que estas transfiram informações como áudio, imagens e dados em uma conexão $P 2 P$ segura [Sredojev et al. 2015]. Para realizar a transferência dessas informações, o WebRTC baseia-se em três API's principais [El Jaouhari et al. 2017]: MediaStream, RTCPeerConnection e RTCDatachannel.

A API RTCDatachannel foi utilizada na implementação do CareLoc para compartilhar as informações de localização entre os pares. Para que a conexão possa ser estabelecida é necessária a presença de um servidor de sinalização, responsável por realizar a negociação entre os pares bem como cuidar do processo de configuração, controle e finalização de uma sessão de comunicação. A Figura 1 ilustra a arquitetura do processo de negociação entre dois dispositivos, organizado em 5 passos.

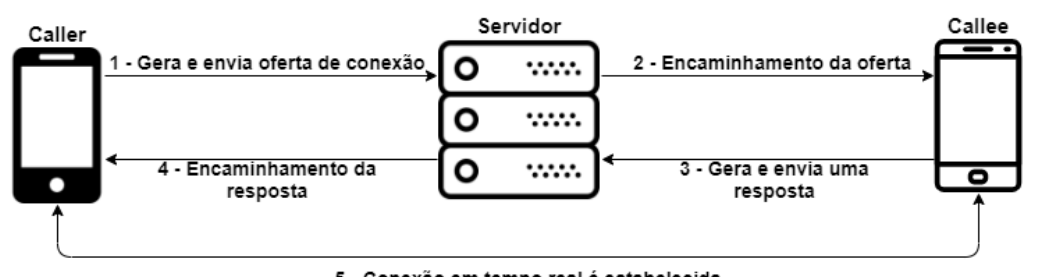

Figura 1. Arquitetura de negociação entre os pares.

Assim que a conexão é estabelecida, o canal de dados pode ser utilizado pela API RTCDatachannel, e, a partir dela, a localização compartilhada com o outro par na forma de dados.

\subsection{Trabalhos relacionados}

A principal inspiração para este artigo foi o sistema apresentado em [Oliveira and Carvalho 2017], que utiliza a técnica de trilateração para realizar a localização. Os trabalhos [Cay et al. 2017, Mussina and Aubakirov 2018, Thaljaoui et al. 2015, Wang et al. 2015, Kuxdorf-Alkirata and Brückmann 2016] também utilizam a técnica de trilateração. Diferentemente, os trabalhos [Li et al. 2016, Filípek and Kovarova 2016, Peng et al. 2016, Tabbakha et al. 2017] implementam a localização por meio da técnica de reconhecimento de padrões e os trabalhos [Hou and Arslan 2017, Kyritsis et al. 2016] aplicam métodos probabilísticos. Por fim, os artigos [Memon et al. 2017, Kyritsis et al. 2016] usam um método baseado em proximidade para a realização da localização. O método utilizado pela solução CareLoc também é um método de proximidade, se assemelhando ao método proposto por [Kyritsis et al. 2016], que divide o ambiente a ser analisado em cômodos, colocando no centro de cada cômodo um beacon para representá-lo e assim realizar a localização. Já o trabalho [Memon et al. 2017], além de dividir o ambiente em cômodos, também divide os cômodos em subespaços menores e atribui a cada um destes subespaços um beacon que o representa. Após o posicionamento dos beacons, é selecionado aquele que possui o maior valor de RSSI, sendo este o escolhido para representar a posição do usuário.

\section{CareLoc: Aplicação de localização}

O desenvolvimento da solução CareLoc empregou o método baseado em proximidade para a realização da localização, escolhido por ser de implementação simples e por exigir uma quantidade mínima de beacons. Um aplicativo Android foi desenvolvido como aplicação de localização. Este aplicativo foi escrito em Java e está disponível em https://github. $\mathrm{com} / \mathrm{matheusntg}$ (assim como as outras aplicações do CareLoc). Antes de realizar a 
localização, a aplicação necessita realizar uma configuração inicial do ambiente em que irá funcionar. Esta configuração existe para coletar os dados que serão utilizados como valores de referência no algoritmo de localização.

Terminada a configuração, a aplicação cria dois objetos representando a casa, sendo um utilizado para o funcionamento da própria aplicação, e o outro convertido para um objeto JSON e enviado para a base de dados do Firebase $^{1}$ a fim de criar a persistência dos dados obtidos. Uma vez que este processo é concluído, a aplicação começa a mostrar ao usuário a sua própria localização. Isto é obtido com a comparação dos valores $R S S I$ que estão sendo recebidos pelos beacons $B L E$ e os valores de referência definidos pela aplicação no momento da configuração.

\subsection{Aplicação de Monitoramento}

A aplicação de monitoramento tem como objetivo informar aos interessados no cuidado, a localização em tempo real de um paciente que deseje ser monitorado. Para realizar este monitoramento só é necessário fornecer um nome de usuário e o nome do paciente que deseja localizar. Assim que estes dados são fornecidos, são enviados ao servidor de sinalização que encaminha as mensagens e realiza a conexão entre os dois pares. Quando a conexão é estabelecida entre os pares, as mensagens (objetos JSON informando a localização) são enviadas em tempo real da aplicação de localização para a de monitoramento.

\subsection{Servidor de sinalização}

O servidor de sinalização tem como objetivo estabelecer a conexão em tempo real entre os pares na rede. Este, se comunica diretamente com a aplicação de localização e a de monitoramento e possui o papel de intermediador entre as partes, transmitindo as ofertas e respostas geradas pelos pares que ainda não estabeleceram uma conexão em tempo real.

\section{Ambiente de testes}

A Figura 2 apresenta o ambiente utilizado para a realização de testes da solução CareLoc, assim como a distribuição dos beacons BLE.

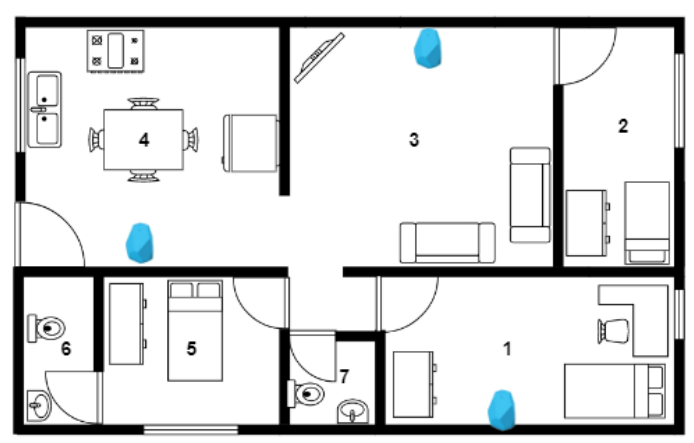

Figura 2. Planta baixa do ambiente de testes utilizado.

Somente os cômodos 1, 3 e 4, respectivamente, sala, quarto e cozinha, abrigam beacons $B L E$ (em azul). Os testes foram realizados utilizando a aplicação de localização para configurar o ambiente e mensurar a posição que a aplicação identificava quando o usuário se posicionava no centro de cada cômodo da casa. Os resultados obtidos são mostrados Tabela 1.

\footnotetext{
${ }^{1}$ https://firebase.google.com/
} 


\begin{tabular}{|c|c|}
\hline Posição real do usuário & Posição identificada pelo aplicativo \\
\hline 1 e 2 & Quarto \\
\hline 3 & Sala \\
\hline $4,5,6$ e 7 & Cozinha \\
\hline
\end{tabular}

Tabela 1. Resultados obtidos pela aplicação

Nota-se que a aplicação sempre identifica a posição correta do usuário quando o mesmo se encontra em um cômodo com o beacon. Quando o usuário se movimenta para um cômodo sem o beacon, a aplicação tende a identificar o cômodo com o sinal de potência mais forte, ou, em outras palavras, o cômodo que possui um beacon de referência mais próximo da posição atual do usuário.

As falhas geradas pela aplicação na identificação da posição do usuário ocorrem pelo fato de um beacon estar diretamente associado a um cômodo e pela possibilidade dos sinais RSSI passarem por obstáculos, como paredes. Isso produz a identificação de posições falsas como as mostradas na Tabela 1, pois, ao ser posicionado em um cômodo que não possui um beacon mapeando aquele espaço, a aplicação identifica os sinais que estão ao alcance dela e filtra o sinal com a maior potência, que em condições estáveis é o sinal do beacon que mapeia para o cômodo mais próximo em que o dispositivo móvel, com o usuário, se encontra. Uma possível forma de lidar com essa limitação é a aplicação de beacons de referência em todos os ambientes da casa, possibilitando uma cobertura total da casa pela aplicação.

\section{Conclusão}

Esse artigo apresentou uma solução para o problema de localização indoor no monitoramento remoto de pacientes utilizando BLE e Real Time Communication com WebRTC. Como parte da pesquisa, uma revisão sistemática foi conduzida [Rodrigues and Carvalho 2019] a fim de entender como se encontra o estado da arte dos métodos de localização indoor utilizando $B L E$, e a partir desta revisão um método de localização foi escolhido para criar a aplicação da solução com base no objetivo da pesquisa.

Durante a condução da pesquisa, notou-se que localizar um usuário em um ambiente dinâmico mantendo a estabilidade da precisão é um problema que não está bem resolvido no meio acadêmico-científico [Rodrigues and Carvalho 2019].

Para trabalhos futuros, a criação de uma aplicação capaz de identificar um usuário como um ponto em um sistema de coordenadas, aumentando assim a precisão, seria essencial. Além disso, o aplicativo criado para o monitoramento, bem como o de localização, é capaz de estabelecer somente uma conexão $P 2 P$ de 1:1. Em ambientes reais, a presença de um sistema capaz de lidar com conexões $R T C$ n:n é indispensável.

\section{Referências}

Cay, E., Mert, Y., Bahcetepe, A., Akyazi, B. K., and Ogrenci, A. S. (2017). Beacons for indoor positioning. In 2017 Intern. Conf. on Engineering and Tech. (ICET), pages 1-5. IEEE.

Del Duca, G. F., Thumé, E., and Hallal, P. C. (2010). Prevalência e fatores associados ao cuidado domiciliar a idosos. volume 45, pages 113-120. SciELO Public Health.

Dickinson, P., Cielniak, G., Szymanezyk, O., and Mannion, M. (2016). Indoor positioning of shoppers using a network of bluetooth low energy beacons. In 2016 IPIN, pages 1-8. IEEE.

El Jaouhari, S., Bouabdallah, A., Bonnin, J.-M., and Lemlouma, T. (2017). Toward a smart health-care arch. using webrtc and wot. In World Conf. on Inf. Sys. and Tech., pages 531-540. Springer. 
Filípek, P. and Kovarova, A. (2016). Indoor loc. based on beacons and calculated by particle filter. In Proc. of the 17th CompSysTech'16, pages 269-276, New York, NY, USA. ACM.

Hou, X. and Arslan, T. (2017). Monte carlo localization algorithm for indoor positioning using bluetooth low energy devices. In 2017 Int. Conf. on Localization and GNSS, pages 1-6. IEEE.

IBGE. Projeção da população do brasil e das unidades da federação. Disponível em: https://www.ibge.gov.br/apps/populacao/projecao/. Acessado em: 25 jun. 2019.

Keele, S. et al. (2007). Guidelines for performing systematic literature reviews in software engineering. Technical report, Technical report, Ver. 2.3 EBSE Technical Report. EBSE.

Kuxdorf-Alkirata, N. and Brückmann, D. (2016). Reliable and low-cost indoor localization based on bluetooth low energy. In 2016 3rd IDAACS-SWS, pages 92-96. IEEE.

Kyritsis, A. I., Kostopoulos, P., Deriaz, M., and Konstantas, D. (2016). A ble-based probabilistic roomlevel localization method. In 2016 ICL-GNSS, pages 1-6. IEEE.

Le, X. H. B., Di Mascolo, M., Gouin, A., and Noury, N. (2008). Health smart home for elders-a tool for automatic recognition of activities of daily living. In 2008 30th Annual International Conference of the IEEE Engineering in Medicine and Biology Society, pages 3316-3319. IEEE.

Li, X., Xu, D., Wang, X., and Muhammad, R. (2016). Design and implementation of indoor positioning system based on ibeacon. In 2016 ICALIP, pages 126-130. IEEE.

Memon, S., Memon, M. M., Shaikh, F. K., and Laghari, S. (2017). Smart indoor positioning using ble technology. In 2017 4th IEEE ICETAS, pages 1-5. IEEE.

Mussina, A. and Aubakirov, S. (2018). Rssi based bluetooth low energy indoor positioning. In 2018 IEEE 12th AICT, pages 1-4. IEEE.

Nourizadeh, S., Deroussent, C., Song, Y.-Q., and Thomesse, J.-P. (2009). A Distributed Elderly healthcare System. In MobiHealth 2009, Porto, Portugal.

Oliveira, A. P. and Carvalho, S. (2017). Localização usando beacons em ambientes internos para monitoramento remoto de pacientes. In Anais V ERI-GO, pages 160-173.

Peng, Y., Fan, W., Dong, X., and Zhang, X. (2016). An iterative weighted knn (iw$\mathrm{knn}$ ) based indoor localization method in bluetooth low energy (ble) environment. In 2016 UIC/ATC/ScalCom/CBDCom/IoP/SmartWorld, pages 794-800. IEEE.

Rodrigues, M. S. and Carvalho, S. T. (2019). Sistemas de localização indoor utilizando bluetooth low energy: Uma revisão sistemática. In SBSI, WICSI, pages 5 - 8.

Sredojev, B., Samardzija, D., and Posarac, D. (2015). Webrtc technology overview and signaling solution design and implementation. In 2015 38th MIPRO, pages 1006-1009. IEEE.

Tabbakha, N. E., Tan, W.-H., and Ooi, C.-P. (2017). Indoor location and motion tracking system for elderly assisted living home. In 2017 ICORAS, pages 1-4. IEEE.

Thaljaoui, A., Val, T., Nasri, N., and Brulin, D. (2015). Ble localization using rssi measurements and iringla. In 2015 ICIT, pages 2178-2183. IEEE.

Trinugroho, Y. B. D., Reichert, F., and Fensli, R. (2012). ehealth smart home environment service platform-enabling remote monitoring and service composition through social media. In Inter. Conf. on Health Inf., volume 2, pages 434-438. SCITEPRESS.

Trinugroho, Y. B. D., Reichert, F., and Fensli, R. W. (2011). A soa-based health service platform in smart home environment. In 2011 IEEE 13th International Conference on e-Health Networking, Applications and Services, pages 201-204. IEEE.

Wang, Y., Ye, Q., Cheng, J., and Wang, L. (2015). Rssi-based bluetooth indoor localization. In 2015 11th International Conference on Mobile Ad-hoc and Sensor Networks (MSN), pages 165-171. IEEE. 